Voix et Images

volxetimages

\title{
Le théâtre québécois : stimulation ou communication?
}

\section{Louis Francoeur}

Volume 1, numéro 2, décembre 1975

\section{Raoul Duguay}

URI : https://id.erudit.org/iderudit/290077ar

DOI : https://doi.org/10.7202/290077ar

Aller au sommaire du numéro

Éditeur(s)

Les Presses de l'Université du Québec

ISSN

0318-9201 (imprimé)

1705-933X (numérique)

Découvrir la revue

Citer cet article

Francoeur, L. (1975). Le théâtre québécois : stimulation ou communication?

Voix et Images, 1(2), 220-240. https://doi.org/10.7202/290077ar d'utilisation que vous pouvez consulter en ligne.

https://apropos.erudit.org/fr/usagers/politique-dutilisation/ 


\section{Le théâtre québécois: stimulation ou communication?}

L'intérêt nouveau que suscite le théâtre québécois depuis quelques années auprès d'un public nombreux et varié, ici même aussi bien qu'à l'étranger, étonne souvent la critique officielle. Le public, lui, comme toujours au théâtre, ne s'y est pas trompé. Et s'il lui est devenu fidèle, c'est peut-être qu'au-delà de l'identification; du divertissement, de l'imitation qu'il lui permet, il y perçoit la communication d'un "message» qui lui est destiné, qu'il reçoit et auquel il répond, à sa façon c'est-à-dire par sa présence assidue, par ses pleurs et ses rires, par ses applaudissements, voire dans certains cas par ses paroles et par ses gestes. Certaines représentations de Medium Saignant, de Double Jeu ou des Belles-Sœurs, sans parler des spectacles plus récents du Théâtre du Même Nom ou du Grand Cirque Ordinaire, nous ont habitués à ce genre d'échanges entre le public québécois et son théâtre.

Ce phénomène de la communication théâtrale au Québec constitue en même temps une double invitation pour le critique d'ici: d'une part élaborer un modèle théorique qui en rendra possible l'analyse et de la façon la plus rigoureuse qui soit, d'autre part, analyser cette polysémie pour en dégager les structures signifiantes. Car si le théâtre peut prendre place aujourd'hui parmi les moyens de communication de masse, il doit faire l'objet d'autant d'attention que tous les autres "procédés de communication, c'est-à-dire des moyens utilisés pour influencer autrui et reconnus comme tels par celui qu'on veut influencer ${ }^{1} »$. Or, c'est précisément comme étant l'étude de ces procédés qu'Éric Buyssens définissait la sémiologie dans son ouvrage sur la Communication et l'articulation linguistique, précisant la pensée de Saussure qui la définissait dans son Cours comme la "science qui étudie la vie des signes au sein de la vie sociale ${ }^{2}$ ".

Ces considérations préliminaires nous amènent à faire trois propositions: premièrement, que le théâtre québécois, comme toute autre représentation théâtrale, est système de signes et processus intentionnel de

1. Éric Buyssens, la Communication et larticulation linguistique, Bruxelles, Presses Universitaires de Bruxelles, 1970, p. 11.

2. Ferdinand de Saussure, Cours de linguistique générale, Paris, Payot, 1969, p. 33 
communication et non seulement moyen de signification; deuxièmement, que la sémiologie de la communication, dans la mesure où elle peut être considérée comme suffisamment fondée, fournira les éléments de description et d'analyse requis par la communication théâtrale; troisièmement, que le recours à un modèle théorique s'impose dès lors que nous prétendons rendre compte d'un processus particulier qui n'est, en somme, que la manifestation singulière d'un processus plus général et plus abstrait.

Cependant, avant d'aller plus loin, il peut être opportun de rappeler le mot de Jakobson sur le désaccord qui règne souvent au sein de la communauté des chercheurs. "Fort heureusement, écrit-il, les conférences scientifiques et politiques n'ont rien de commun. Le succès d'une convention politique dépend de l'accord de la majorité ou de la totalité de ses participants. En revanche, le recours au vote et au veto est étranger aux débats scientifiques, où le désaccord se révèle en général plus productif que l'accord ${ }^{3}$. " C'est dans cet état d'esprit que nous avons entrepris notre travail qui, on le verra, nous place en désaccord avec un certain nombre de chercheurs, notamment Georges Mounin qui écrivait dans son Introduction à la sémiologie "qu'on explique mieux ce qui se passe au théâtre en termes de “stimulation » qu'en termes de communications ${ }^{4}$ ».

Pourtant, si l'on y regarde de près, c'est-à-dire en considérant les facteurs constitutifs de tout procès linguistique, de tout acte de communication verbale, identifiés par R. Jakobson dans ses Essais de linguistique générale ${ }^{5}$, on peut dégager de l'œuvre dramatique des facteurs constitutifs qui sont étroitement apparentés aux premiers. On y retrouve, en effet, le destinateur (ou l'encodeur) qui est le personnage-acteur parlant qui envoie un message au destinataire (ou décodeur) qui est au niveau intradiégétique le personnage-acteur écoutant et au niveau extradiégétique "le relais fictif " du spectateur assis dans la salle. Pour être transmis et perçu, ce message requiert un contexte auquel il renvoie et qui sera, au théâtre, verbal ou scénique ou une combinatoire des deux groupes d'éléments référents; le message requiert également un code commun au destinateur et au destinataire, code qui au théâtre sera constitué de tous les signes du langage dramatique, qu'ils soient verbaux, gestuels ou scéniques. Enfin, toujours selon Jakobson, le message requiert un contact, un canal physique et une connexion psychologique entre le destinateur et le destinataire qui leur permettent d'établir et de maintenir la communication. C'est trop peu de dire que ce contact existe dans la dramaturgie. Sans lui, point de spectacle, point de re-présentation.

Loin donc de n'être qu'un moyen de stimulation du spectateur, le théâtre se présente à nous avec tous les caractères d'un véritable processus intentionnel de communication qui fonctionne selon un système de signes. Que le langage théâtral soit composé de signes et non uniquement de symboles, cela apparaîtra à l'examen du caractère arbitraire, linéaire et

3. Roman Jakobson, Essais de linguistique générale, tome I, Paris, Minuit, 1963, p. 209.

4. Georges Mounin, Introduction à la sémiologie, Paris, Minuit, 1970, p. 92.

5. Roman Jakobson, op. cit., p. 213. 
discret des éléments qui le composent. Il n'est pas douteux, en effet, que des costumes, des décors, des gestes, des noms de personnages ne manifestent aucune motivation entre leur signifiant et leur signifié et peuvent être considérés comme des signes. De même, s'il y a simultanéité au théâtre entre certains éléments, il existe aussi des combinaisons liées au caractère irréversible du temps qui indiquent bien que l'énoncé dramatique est aussi une suite de signes «émis dans le temps et perçus dans le temps ${ }^{6}$ ". Enfin, lié au caractère linéaire du signe apparaît son caractère discret ou différentiel, "propriété de pouvoir être distingué par oui ou par non ${ }^{7}$. Comme les signes linguistiques, les signes dramatiques sont habituellement ou semblables ou différents. Le décodage du message auquel le spectateur est invité à participer l'exige.

C'est donc dans beaucoup de cas à d'authentiques signes que nous avons affaire au théâtre et à des signes qui sont des "unités bien définies qui se combinent ou se structurent selon des règles définies ou connues ${ }^{8}$ ", en d'autres mots, des unités qui appartiennent à un système. Nous aurons l'occasion de préciser quelles sont ces règles. Mais dès maintenant nous pouvons avancer que le théâtre, système de signes, processus intentionnel de communication se distingue des “moyens" de communication ou de signification a-systématiques que sont «les arts plastiques, l'affichage publicitaire, les enseignes ${ }^{9}$, la mode ou l'art culinaire. A ce titre, il peut être considéré comme un phénomène qui relève d'une sémiologie de la communication et non seulement d'une sémiologie de la signification telle que décrite par R. Barthes dans ses Éléments de sémiologie ${ }^{10}$. Ce sont donc les concepts propres à la sémiologie de la communication qui seront utilisés ici dans l'élaboration d'un modèle théorique de description de l'œuvre dramatique. Car s'il n'est de science que du général et d'objets que singuliers, toute analyse qui vise à rendre compte rigoureusement d'un système, d'une structure particulière, doit prendre appui sur un modèle théorique préexistant à l'œuvre qu'il s'agit de décrire. Ce modèle, «représentation symbolique d'un processus dont on essaie de rendre compte ${ }^{11}$ " n'est pas comme on le pense souvent un carcan que l'on impose au spectateur ou au lecteur de l'œuvre, c'est, au contraire, un guide sûr dans la découverte de ses structures les plus profondes et un instrument qui permet de l'identifier et de la décrire.

Nous esquisserons à grands traits ce que pourrait être ce modèle théorique, synthèse des recherches actuelles en sémiologie, en recourant le plus souvent possible à des applications que nous avons faites dans le théâtre québécois.

6. Ferdinand de Saussure, op. cit., p. 103.

7. Georges Mounin, op. cit., p. 73.

8. Ibid., p. 71.

9. Ibid.

10. Roland Barthes, "Éléments de sémiologie", Communications, n 4 (1964), p. 91 144.

11. On pourrait retenir cette autre définition: «Représentation simplifiée d'un processus, d'un système.» 


\section{Le titre}

En premier lieu, nous avons à nous interroger sur le titre. La relation du titre avec la pièce en est une d'opposition. Le titre s'oppose à la pièce comme le nom à la phrase (Florence, Ben Ur, la Duchesse de Langeais), comme le sommaire au récit (Encore cinq minutes, T'es pas tannée Jeanne d'Arc?), comme le signifiant au signifié (Wouf! Wouf!, Au retour des oies blanches). Par cette opposition, le titre nomme, résume, oriente l'action dramatique qui va suivre ${ }^{12}$.

\section{Diviser le texte dramatique en unités minimales}

En deuxième lieu, si ce n'est déja fait par l'auteur lui-même, ił faudra découper le texte dramatique en unités minimales. L'analyse est articulation du texte en unités discrètes ${ }^{13}$. Nous pourrons retenir comme critères de découpage ceux qu'a proposés Steen Jansen ${ }^{14}$, reprenant en cela la pratique des auteurs du xvile siècle: en empruntant au plan scénique les entrées ou sorties de personnages, les changements de décor. Chaque unité que nous proposons d'appeler séquence est simultanément un élément intégré d'une unité plus grande (l'œuvre dramatique) et un intégrant d'unités plus petites à l'intérieur de sa totalité (les «fonctions»). En tant qu'intégrée, la séquence assure ou n'assure pas la cohérence de l'œuvre comme système de signes, en tant qu'intégrante elle permet ou non la génération des constituants du récit-drame, les «fonctions".

Ainsi, le découpage du texte dans Encore cinq minutes nous permet d'identifier 9 séquences dans le premier tableau et 11 dans le deuxième. On y retrouve donc sensiblement le même équilibre entre les tableaux que dans les œuvres classiques de même dimension.

\section{Identifier les signes initiaux et les signes finaux}

En troisième lieu, nous identifions les signes initiaux et les signes finaux du système/processus dramatique. "La tâche d'une description scientifique, c'est d'expliquer comment s'effectue le passage de A à B et quelles sont les liaisons entre ces deux ensembles ${ }^{15}$. " Un exemple choisi entre plusieurs suffira à la démonstration. Dans Encore cinq minutes, nous pouvons identifier les signes initiaux suivants: "Une pièce blanche et vide où Gertrude tourne en rond. Sur la surface lisse d'un mur, une lézarde se creuse, s'allonge, pousse des rameaux, petites fissures quasi imperceptibles qui bientôt envahiront tout le mur..." Un seul personnage, Gertrude: «Plainte angoissée. Gémissement oppressé. - «Quoi faire?" - «Quoi ?».

12. Louis Marin, Etudes sémiologiques, Paris, Klincksieck, 1971, p. 250.

13. Émile Benveniste, «Sémiologie de la langue», Semiotica, La Haye, Mouton, vol. I (1969), n' 1 , p. $1-12$ et $n^{\circ} 2$, p. 127-135.

14. Steen Jansen, “Esquisse d'une théorie de la forme dramatique», Langages, $n^{\circ} 12$ (décembre 1968).

15. I. I. Revzin, «les Principes de la théorie des modèles en linguistique», Langages, n०15 (septembre 1969), p. 28. 
Une question est donc posée par les signes initiaux. Qu'en est-il maintenant des signes finaux? "Même pièce mais envahissement d'objets (meubles, bibelots) hétéroclites et dans un grand désordre. " Quatre personnages sont en scène, dont Gertrude qui s'apprête à quitter la pièce et les siens. L'ensemble des signes de la dernière séquence apporte la réponse à la question posée dans la première. Comment sommes-nous passés de la question à la réponse? La description que permet le modèle théorique a précisément pour objet de nous le révéler.

\section{4. et 5. Inventaire et classement des signes dramatiques}

Avant tout effort d'analyse, un inventaire complet et un classement de tous les signes du langage dramatique s'imposent d'emblée. Aucun ne doit être laissé de côté, car à priori, pour l'analyste, ils sont tous de même valeur. Seul leur rôle dans la génération des «fonctions" permettra de les distinguer et d'établir entre eux une hiérarchie. Plusieurs façons de classer les signes peuvent être retenues. Cependant, il nous semble que tout classement devrait tenir compte du caractère hétérogène des signes dramatiques, certains relevant du niveau proprement textuel comme les répliques et les indications de régie, d'autres au contraire appartenant plus spécifiquement au niveau scénique comme les personnages et les décors. On devra également, dans le classement, retenir l'aspect permanent de certains signes dramatiques et l'aspect transitoire de certains autres. A titre d'illustration, on pourra consulter le tableau I.

Déjà cet inventaire et ce classement fournissent à l'analyste des données qui parfois peuvent être précieuses. Ainsi, le seul classement des signes dans Encore cinq minutes nous permet, entre autres, de constater que la proportion entre le nombre des répliques et leur étendue (1153 répliques pour une étendue de 1911 lignes) indique que, dans l'ensemble de la pièce, le dialogue sera vif et que les longs échanges seront rares; ou bien encore que le seul personnage jouissant d'une autonomie scénique est Gertrude et qu'en contrepartie son mari, Henri, n'apparaît jamais seul en scène avec l'un ou l'autre de ses enfants.

\section{Tableau des signes dramatiques}

Tous les signes dramatiques apparaîtront dans un tableau établi à partir des indications de régie. (Voir tableau la)

\section{7. Écrire les graphes arborescents}

De même que la langue, selon une certaine tendance de la linguistique moderne, ne devrait être disjointe de la parole, de même la dramaturgie ne devrait être séparée de telle représentation particulière ou de tel texte dramatique singulier. II y aurait donc avantage à analyser le proces- 
sus-système de tel texte dramatique comme la linguistique trouve son profit à analyser le processus de l'énonciation linguistique ${ }^{16}$.

En utilisant les notions chomskyennes de compétence et de performance, nous poserons comme hypothèse que la compétence d'un auteur dramatique peut se définir comme la somme des connaissances qui le rendent apte, au niveau de la performance, à créer des textes dramatiques, à la limite en nombre infini. Ces connaissances sont essentiellement des aptitudes à utiliser des procédés, à jouer avec des règles. Selon quelles lois ou règles le dramaturge est-il parvenu à faire de tous ces signes dramatiques hétérogènes un spectacle-récit, une performance? Répondre à cette question, ce serait connaître la véritable compétence de l'auteur dramatique. Autrement dit, en dehors et au-delà de la structure superficielle des réalisations concrètes de la performance, existe-t-il une structure profonde du langage dramatique dont chaque dramaturge se servirait au moment de la création de l'œuvre? Nous serions alors dans les limites d'une “grammaire" du récit dramatique qui devrait comprendre un ensemble fini de règles susceptibles de générer un nombre infini de récits dramatiques. Nous proposons de considérer la «logique des possibles narratifs" élaborée par Claude Bremond ${ }^{17}$ comme cet ensemble fini de règles récursives capables d'engendrer les “phrases-noyaux» dont les prédicats deviendront les «fonctions" du récit au sens proppien du terme.

Cette «grammaire» devra fournir à chaque phrase-fonction une description univoque et structurale qui a déjà été formalisée par Chomsky sous la forme d'un graphe arborescent auquel on a donné le nom d'indicateur syntagmatique et dont le principe a été énoncé par $\mathrm{N}$. Ruwet de la façon suivante: “l'arbre a un certain nombre de nœuds étiquetés (SN, SV); on dira que tous les éléments qui peuvent être rattachés à un certain riœud sont un constituant du type désigné par l'étiquette qui appelle le nœud ${ }^{18}$ ". Ainsi donc, des signes qui seraient contigus mais qui ne pourraient être greffés à un même nœud, ne pourraient faire partie du même constituant ${ }^{19}$. (Voir le tableau Ib)

Nous proposons de considérer les signes, tous les signes, qui apparaissent dans le tableau par ordre d'indication de régie comme susceptibles de jouer un rôle ou de déterminant ou de nom ou de prédicat dans la phrase-fonction. Il s'agit d'en connaître le signifié pour savoir à quel nœud les rattacher comme constituants. Nous aurons alors un nombre de phrases égal au nombre de fonctions dans la pièce. Ces fonctions s'organisent à leur tour selon une certaine logique que Propp, Bremond et Greimas ont étudiée dans leurs travaux de sémiologie. Nous avons opté pour le modèle triadique de Claude Bremond.

16. Voir J. Peytard et E. Genouvrier, Linguistique et enseignement du français, Paris, Larousse, 1970, p. 122.

17. Claude Bremond, "la Logique des possibles narratifs", Communications, $n^{\circ} 8$ (1966), p. 60-76.

18. Nicolas Ruwet, Introduction à la grammaire générative, Paris, Plon, 1968.

19. Noam Chomsky, Structures syntaxiques, Paris, Seuil, 1969, p. 29-55. 


\section{Schéma de la logique des fonctions}

Rappelons seulement ici quelques notions fondamentales de l'étude de Bremond. L'univers raconté, l'histoire ou la diégèse, est régi par des lois qui sont articulées doublement par les contraintes logiques que tout récit, série d'événements, pour être intelligible doit respecter et par les conventions propres à chaque «genre littéraire». L'unité de base est la fonction au sens proppien du terme, action ou événement, qui, organisée en triade, engendre un récit. La triade correspond aux trois phases obligées de tout processus, analogiquement comparable à l'expansion d'une forme verbale, infinitif-participe présent-participe passé: une fonction qui ouvre la possibilité du processus, une fonction qui réalise cette virtualité, une fonction qui clôt le processus sous forme de résultat atteint. Ces triades, enfin, s'enchaînent selon un cycle narratif normatif pour constituer ce que nous sommes convenus d'appeler depuis l'étude de Benveniste ${ }^{20}$ le niveau de l'histoire. (Voir tableau Ic)

\section{Sémiologie du personnage}

La connaissance des fonctions du récit nous conduit à l'étape suivante de la description de l'œuvre dramatique qui est la connaissance de ceux qui assument ces fonctions, les personnages-acteurs. L'étude des personnages-acteurs s'impose d'autant plus que l'on peut considérer que tout le récit-drame trouve en eux son support et qu'à toute fin utile, ils sont le seul domaine où l'on atteigne le "contenu de l'énoncé " comme l'a souligné Lévi-Strauss dans son article «la Structure et la forme ${ }^{21}$ ». Pour préciser cette partie de notre étude, nous ferons appel aux travaux récents de François Rastier, Sorin Alexandrescu, Lévi-Strauss, A.-J. Greimas et plus particulièrement à l'article de Philippe Hamon sur le statut sémiologique du personnage ${ }^{22}$. Nous prendrons comme corpus exemplaire la pièce de Marcel Dubé, Au retour des oies blanches.

Il serait trop long dans le cadre de cet exposé de fonder dans le détail la pertinence de l'analogie entre le personnage dramatique et le signe. Nous pouvons tout au plus reprendre l'énumération d'un certain nombre de caractères propres aux langues naturelles et qu'il nous semble ressortir également, mutatis mutandis, au langage dramatique et à ses signes-personnages: le théâtre est processus intentionnel de communication, système de signes dans lequel on retrouve la linéarité qui permet d'identifier des suites de signes émis dans le temps et perçus dans le temps, dotés d'un caractère discret et souvent arbitraire.

20. Émile Benveniste, Problèmes de linguistique générale, tome 1, Paris, Gallimard, 1966, p. 238.

21. Claude Lévi-Strauss, "la Structure et la forme», Cahiers de l'Institut de science économique appliquée, Paris, ISEA, $n^{\circ} 9$ (mars 1960), série $M n^{\circ} 7, p .3-36$.

22. Philippe Hamon, «Pour un statut sémiologique du personnage», Litterature, $n^{\circ} 6$ (mai 1972). 
Le classement suggéré par Hamon en personnages référentiels, déictiques et anaphores sur le modèle des trois subdivisions reconnues par plusieurs sémiologues, savoir la sémantique, la syntaxe et la pragmatique nous permet, en premier lieu, de découvrir Achille, le père, comme personnage référentiel et comme personnage anaphore. Référentiel en tant qu'il appartient à une catégorie sociale bien déterminée, celle des politiciens malhonnêtes, référentiel également par son prénom. La référence à la mythologie deviendra explicite dans la bouche de son fils, Robert, qui a donné comme titre au roman qu'il achève d'écrire: le Talon d'Achille et qui le résume ainsi: "Je raconte l'histoire d'un homme que sa mère a voué à la grandeur et qui se rend compte à cinquante ans qu'il a hérité de toutes les faiblesses et de toutes les tares paternelles. "Achille est aussi un personnage anaphore par son rôle de mémoire dans la pièce, rappelant certains faits de son propre passé tout aussi bien que de celui de sa femme, Élizabeth, et de son demi-frère, Tom; anaphore également par les faux indices qu'il sème pour tromper son entourage et plus particulièrement sa fille Geneviève; anaphore, enfin, par les faux projets qu'il met de l'avant quant à son avenir personnel et à celui de sa famille. Parmi tous les personnages de la pièce, Achille est celui que le classement nous présente comme le support du plus grand nombre de marques.

Analogon du signe, le personnage doit être décrit à la fois comme signifiant et comme signifié.

Le signifiant du personnage du père dans $A u$ retour des oies blanches est désigné par 47 constituants différents qui forment un paradigme grammaticalement hétérogène puisque nous $y$ retrouvons aussi bien les pronoms je-me-moi, tu-vous-il que le syntagme «mon cher petit papa dégonflé » et un paradigme sémiologiquement hétérogène dans lequel apparaissent ses deux costumes, ses gestes et ses paroles. L'ordre d'apparition des constituants du signifiant du père, leur distribution dans la pièce et leur récurrence révèlent la transformation profonde que subit en même temps le signifié. En effet, le costume tout à fait correct et traditionnel qu'il porte durant la majeure partie de la pièce est remplacé au dernier tableau par un "costume de bouffon", tandis que les termes neutres de «ton père» ou «papa» sont écartés au profit d'expressions du type de «sale petit tricheur de la pire espèce». A cet égard, nous pouvons affirmer que la «grammaire» du signifiant du père est déjà révélatrice de l'opération d'inversion du contenu à laquelle l'étude du signifié nous permettra d'assister.

Notre description du signifié du personnage-signe a pour objet la connaissance du personnage, ici du personnage du père, Achille, non pas tant par les indices caractériels dont il est le support que par les relations qu'il entretient avec les autres personnages tant il est vrai qu'un personnage se définit moins par son essence que par son existence, c'est-à-dire par le réseau complexe des ressemblances et des différences qui seront révélées à l'aide d'un nombre plus ou moins élevé d'axes sémantiques pertinents et récurrents. Le personnage est alors considéré comme un sémème, unité sémantique composée d'un faisceau de traits sémantiques 
appelés sèmes qui auront comme analogons les axes sémantiques. Ainsi dans $A u$ retour des oies blanches, nous avons retenu les axes sémantiques de la motivation psychologique, de l'amour, de la sexualité, de la morale, de la parenté surestimée et sousestimée. Ce sont tous des axes fonctionnels. Nous laissons de côté les axes sémantiques qualificatifs parce qu'ils nous semblent moins importants dans l'économie de notre récit-drame. En d'autre temps, nous pourrions les retenir. (Voir tableau II)

Les axes sémantiques retenus laissent entrevoir que le père, Achille, est un personnage grandement motivé psychologiquement puisqu'il est marqué à la fois par son enfance et par un événement important de sa vie adulte. Cependant, en cela, il n'est pas différent de sa femme Élisabeth et de ses enfants Geneviève et Robert qui appartiennent à la même classe que lui. En revanche, l'axe de l'amour permet de noter une différence importante entre Achille et sa femme d'une part, Achille et sa fille d'autre part. Alors qu'il n'est marqué d'aucune façon, Geneviève l'est en recevant plusieurs qualifications d'amoureuse et en posant plusieurs actes fonctionnels d'amour. Élisabeth, pour sa part, reçoit une qualification d'amoureuse et a posé un acte fonctionnel d'amour. Mais si le père d'Au retour des oies blanches est exclu de l'axe sémantique de l'amour, il se retrouve au même titre que sa femme et sa fille dans l'axe de la sexualité où il apparaît comme un des personnages le plus sexué. On doit songer ici à sa relation avec Laura qui ressemble d'ailleurs à un véritable viol. L'axe de la morale est encore plus révélateur des relations oppositionnelles d'Achille avec sa femme et ses enfants. Non amoureux mais sexué, il se qualifie lui-même plusieurs fois selon les règles de la morale conventionnelle alors qu'il pose et a posé plusieurs actes qui contreviennent à cette morale, tandis que sa fille, Geneviève, s'oppose à lui par la cohérence de ses qualifications et de ses actes qui tous les deux vont à l'encontre de la morale conventionnelle (par exemple, son aventure avec l'oncle Tom). Enfin, Achille entretient des rapports de parenté surestimés avec sa mère et sous-estimés avec sa femme, ses enfants et son demi-frère Tom. Achille est donc un personnage important dans l'économie du récit-drame puisqu'il est défini par quatre axes sémantiques sur les cinq qui ont été retenus. C'est essentiellement un personnage d'opposition, divisé contre lui-même, où l'on voit poindre le jeu de l'être et du paraître, opposition surtout avec son entourage immédiat à l'exclusion de sa mère.

Pour préciser davantage les relations du personnage-signe, nous pouvons décomposer un certain nombre d'axes sémantiques en traits distinctifs pertinents simultanés à la manière de la linguistique qui considère le phonème comme faisceau de traits pertinents simultanés. Nous aurons recours au modèle constitutionnel que propose Greimas dans Du Sens ${ }^{23}$ pour les jeux des contraintes sémiotiques et nous retiendrons, aux fins d'analyse, les axes sémantiques de la sexualité, de l'amour et de la morale. (Voir tableau III) Les résultats obtenus sont tout à fait révélateurs du comportement d'Achille. Avec sa femme, par exemple, il entretient des relations

23. A.-J. Greimas, Du sens, Paris, Seuil, 1971, p. 135. 
sexuelles socialisées conflictuelles dont le manque est comblé par sa relation avec Laura. Autre exemple, avec sa fille, Geneviève, le père qu'est Achille entretient des relations d'amour conflictuelles fortes dont le manque sera comblé par sa relation avec sa propre mère, Amélie.

Unité distinctive et signifiante dans le réseau des relations révélé par le modèle constitutionnel, Achille se définit de plus en plus comme un fils soumis, un mari infidèle et un père indigne. II peut être encore défini plus précisément "par son mode de relation avec un lexique de personnages-types beaucoup plus généraux, les actants ${ }^{24}$ ". Que ce soit sous une forme fonctionnelle comme chez Propp ou sous la forme des signes astrologiques comme chez Souriau ou encore selon la nomenclature proposée par Greimas, nous nous retrouvons dans tous les cas en présence d'un paradigme d'un niveau supérieur d'abstraction qui permettra de connaître quel personnage occupe tel ou tel pôle actantiel dans telle séquence du récit-drame. Nous optons ici pour le modèle actantiel proposé par Greimas dans sa Sémantique structurale ${ }^{25}$. "Le récit étant une combinatoire orientée de classes logiques complémentaires", nous devons en premier lieu découper le récit-drame en séquences, si ce n'est déjà fait. Nous pouvons utiliser le découpage fourni par l'étude préalable de la logique des fonctions ou encore recourir à celui que propose Greimas à la suite de LéviStrauss. Ce qui importe, en définitive, c'est d'obtenir, par le biais du découpage de l'énoncé en séquences, les fonctions majeures exercées par les personnages du récit. Pour les besoins de notre analyse et pour diversifier notre démonstration, nous ferons appel aux critères sémantiques suggérés par Greimas, savoir qu'il peut exister au niveau discursif trois sortes de syntagmes, les syntagmes narratifs performanciels, contractuels et disjonctionnels ${ }^{26}$. Le découpage ainsi pratiqué dans $A u$ retour des oies blanches laisse apparaître Achille, le père, d'abord au sein d'un syntagme disjonctionnel au moment de son arrivée en scène comme héros apparent, puis au sein de deux syntagmes performanciels, le premier où il livre combat à sa femme, le second où il doit affronter Geneviève, sa fille, qui par l'aide qu'elle reçoit de son amie Laura est marquée comme véritable héros. À la fin du premier tableau, de héros apparent qu'il était au début, le père est devenu non-héros. Le deuxième tableau (voir tableau IV) laisse encore apparaître Achille au sein d'un syntagme performanciel puisque son combat avec sa fille se poursuit. Cependant, l'aveu de son échec politique le confirme dans son statut de non-héros. C'est au troisième tableau, à l'intérieur de nouveaux syntagmes performanciels d'épreuve que la défaite du père aux mains de sa fille est achevée. De non-héros, il devient traître. Enfin, au quatrième tableau, après la mort de sa fille, le héros véritable, le pèretraître se retrouve au sein d'un syntagme contractuel où avec sa mère, Amélie, il propose un mandement qui est accepté. La vie va continuer et le traître redeviendra héros apparent.

24. Philippe Hamon, op. cit., p. 104.

25. A.-J. Greimas, Sémantique structurale, Paris, Larousse, 1966.

26. A.-J. Greimas, Du sens, p. 190. 
Ce découpage en syntagmes narratifs a laissé apparaître un certain nombre de fonctions exercées par les personnages-acteurs : retour, mandement, combat, victoire ou défaite. Ce sont ces fonctions qui nous permettront maintenant d'identifier les actants de chaque séquence puisqu'elles sont «créatrices d'actants ${ }^{27}$ ", en même temps qu'elles sont dominées par eux en tant qu'ils représentent un modèle d'organisation d'un niveau hiérarchique supérieur. Achille, présent dans 7 séquences sur 12 apparaît essentiellement au tableau des actants comme l'opposant ( $T$ ) puisqu'il s'y trouve cinq fois et comme destinateur $\left(D_{1}\right)$, mais même dans ce dernier rôle il est encore opposant puisqu'il propose à Élisabeth d'oublier Geneviève et à sa mère de retourner voir le premier ministre pour obtenir ses faveurs. Jamais au cours de la pièce n'est-il sujet (S) ou adjuvant (A), deux rôles éminemment actifs.

En résumé, le personnage du père dans $A u$ retour des oies blanches peut être défini comme référentiel et anaphore, ayant des signifiants qui sont distribués de façon telle qu'ils indiquent une transformation profonde du signifié. Le signifié manifeste à son tour une absence totale d'amour mais une sexualité importante, une conduite morale qui n'est pas conforme aux principes moraux dont le personnage se réclame, une surestimation des rapports avec sa mère et une sous-estimation de ceux qu'il entretient avec sa femme et ses enfants. Ce personnage du père ainsi défini par ses relations apparaît finalement comme un être d'opposition qui subit l'action plutôt qu'il ne la commande. Héros apparent, l'inversion du contenu du récit-drame par la médiation du héros réel qu'est sa fille en fait un antihéros marqué.

Ce sont des conclusions partielles qui pourraient bien être généralisées lorsque notre analyse des données recueillies au cours d'un récent séminaire sur les personnages de pères dans le théâtre de Marcel Dubé sera achevée. Du moins, un bilan provisoire le laisse-t-il entrevoir ${ }^{28}$.

En intégrant l'étude sémiologique du personnage au modèle théorique de description de l'œuvre dramatique, nous avons voulu, au plan théorique, formaliser le projet de Philippe Hamon et simultanément mener à terme une analyse détaillée d'un personnage de père chez Dubé qui révèle l'importance du personnage-signe dans la polysémie dramatique.

\section{0. Établir la cohérence de l'oeuvre dramatique}

L'étape suivante de la description de l'œuvre dramatique consistera à en établir la cohérence. Cette cohérence de l'œuvre sera essentiellement fondée sur la relation qui existe entre les séquences et la présence ou l'absence de fonctions en elles. Nous suivons ici la démarche proposée par Steen Jansen sans toutefois recourir aux mêmes éléments pertinents que

27. A.-J. Greimas, Sémantique structurale, p. 129.

28. Ont participé à ce séminaire donné a l'Université Laval à la session d'hiver 1974: Marie Grenier-Francoeur, Alison Baxter-Walker, Raymond Fleury, Réjean Boutin, Gilbert Tremblay, Jean-Guy Hudon et Jean Vermette. Nous tenons à les remercier pour leur précieuse collaboration. 
lui. II nous semble en effet plus opportun de retenir les fonctions comme critères de cohérence puisque ce sont elles en définitive qui constituent le récit.

Nous avons depuis le début de notre exposé défini l'œuvre dramatique comme processus et comme système. C'est à l'égard de ces deux aspects de l'œuvre que nous en établirons la cohérence. Comme processus, l'œuvre théâtrale présente une suite de séquences qui sont en relation avec leurs voisines immédiates; soit de juxtaposition soit d'antéposition. Pour connaître à quelle relation nous avons affaire, il s'agit de faire jouer la preuve de changement. Si une séquence $B$ ne peut à cause de la fonction ou des fonctions qu'elle renferme être placée avant la séquence $A$, nous dirons qu'elle est dans une relation de dépendance vis-à-vis la séquence $A$ ou d'antéposition. Dans le cas contraire, nous parlerons d'indépendance ou de juxtaposition. Comme système, l'œuvre dramatique présente un ensemble de séquences qui entretiennent entre elles des relations d'indépendance (combinaison), de dépendance (sélection) ou d'interdépendance (solidarité). Pour identifier ces relations des séquences dans le système, il s'agit de faire jouer la preuve d'omission. Si une séquence $B$ ne peut à cause de la ou des fonctions qu'elle renferme être omise sans modifier la ou les fonctions de la séquence $\mathrm{A}$ ou $\mathrm{C}$ ou des deux à la fois, nous dirons que sa relation en est une de dépendance ou d'interdépendance. Dans le cas contraire, nous parlerons de relation d'indépendance.

Ainsi, à partir de ces critères, l'analyse de la cohérence dans la pièce de Dubé, Un matin comme les autres, révèle un processus et un système parfaitement cohérents, tandis que la même analyse dans Pauvre Amour nous met en présence d'un processus et d'un système où les séquences indépendantes sont très nombreuses, particulièrement à cause de l'absence de fonctions. Dans ces derniers cas, nous avons affaire parfois à des séquences vides, parfois à des séquences non fonctionnelles mais indicielles qui ont par conséquent un rôle complémentaire dans l'économie du récitdrame.

\section{1. Étude du Destinataire extradiégétique : son statut}

Dernière étape, enfin, celle qui doit étudier le statut du Destinataire extradiégétique. Que le texte dramatique, sauf dans de rares exceptions qu'il faudrait d'ailleurs réexaminer, n'existe que pour être représenté, cela est facilement admis de tous. II faut en tenir compte dans l'examen du texte dramatique lui-même. Par exemple, les personnages déïctiques, porteparole du Destinataire extradiégétique (ou $D_{2}$ extra.) dans $A u$ retour des oies blanches manifestent clairement la présence de ce spectateur fictif. Ainsi Richard: “Je ne sais toujours pas à quel spectacle j'ai été convié" ou encore: "ll commence à y avoir du suspense! Je dois même avouer que j'y prends goût" ou encore dans la bouche de Robert: “On est dans la tragédie grecque ou dans le vaudeville? Moi, je ne sais plus. " Un dernier exemple, lorsque Richard affirme: «Nous allons peut-être assister à de nouvelles révélations. Restons aux aguets.» Le personnage de Louis, Luigi, 
Ludovic de Pauvre Amour est le personnage-déïctique par excellence puisqu'il s'adresse directement au $\mathrm{D}_{2}$ extra. Louis (avant de sortir, au public): «Que fêteront-ils? Le départ des jeunes gens, ou de nouvelles retrouvailles ? Ils sont seuls. Eux seuls décideront. Moi, je n'ai plus qu'à me taire." (Deuxième scène)

Ce $D_{2}$ extra. n'est pas assimilable aux spectateurs réels, car sauf exception, les spectateurs réels peuvent savoir s'ils sont dans la tragédie ou le vaudeville. Ce $D_{2}$ extra. est donc fictif. Mais il n'est pas davantage le spectateur virtuel imaginé par Dubé puisque déjà après une ou deux générations les spectateurs virtuels se sont transformés et même leurs enfants ne sont plus les mêmes spectateurs que leurs parents. Par exemple, leur viendrait-il à l'esprit aujourd'hui d'opposer la tragédie grecque au vaudeville? Enfin, il ne faudrait pas confondre le $D_{2}$ extra. avec le spectateur idéal puisqu'une intervention comme celle de Richard qui affirme: «Je ne sais toujours pas à quel spectacle j'ai été convié " révèle un spectateur ignorant ou distrait qui est donc loin de ressembler au spectateur idéal que souhaite tout dramaturge.

Le $D_{2}$ extra. est un être de raison fictif qui fait partie du processus intentionnel de communication qu'est le théâtre, qui servira en quelque sorte de relais entre le dramaturge et son public comme les personnages eux-mêmes, autres êtres fictifs. De même que Genette ${ }^{29}$ et plus particulièrement Prince ${ }^{30}$ ont reconnu et décrit le rôle important qu'occupe le narrataire dans le système narratif, de même devons-nous reconnaître le rôle du $D_{2}$ extra. dans le système de signes dramatiques et en décrire le fonctionnement par le biais du modèle théorique. D'ailleurs, l'analogie qui existe entre les deux entités, narrataire et $D_{2}$ extra., sera éclairante pour nous puisque l'étude du premier, déjà sérieusement amorcée par les travaux de Prince, nous fournira des pistes à parcourir pour définir le $D_{2}$ extra., le classer et identifier ses fonctions dans la dramaturgie.

A titre d'exemple, et pour limiter notre étude, le $D_{2}$ extra. des BellesSœurs de Michel Tremblay qui n'est pas le spectateur réel, virtuel ou idéal de Montréal, de Sainte-Justine-de-Dorchester ou de Paris, est invisible mais constamment présent par sa situation dramatique révélée par les nombreuses explications que lui fournit le Destinateur, comme dans le monologue de Germaine Lauzon au téléphone au début de la pièce ou par les comparaisons et métaphores nombreuses qui supposent la connaissance que le $\mathrm{D}_{2}$ extra. en a: (“C'est ben simple, si j'me r'tenais pas, j'braillerais comme une vache!" dira Marie-Ange Brouillette), ou par la technique scénique des chœurs des femmes ou encore par l'usage qui est fait du black-out et des projecteurs sur les personnages qui s'expliquent. Ces procédés dramatiques, qui sont aussi des signes du système, rendent possible la communication du message réservé au $\mathrm{D}_{2}$ extra. dans les Belles-Sœurs, à titre de relais. C'est là sa première fonction.

29. Gérard Genette, Figures III, Paris, Seuil, p. 265.

30. Gérald Prince, "Introduction à l'étude du narrataire», Poétique, n 14 (1973), p. 178-196. 
Cependant ce $D_{2}$ extra. n'est pas que le relais du message. II exerce d'autres fonctions dans la pièce. Par exemple, il peut servir comme le narrataire de Prince à caractériser tel personnage. Ainsi Pierrette Guérin, la fille de cabaret, se définit autant par les relations qu'elle entretient avec le $D_{2}$ extra. dans son monologue que par ses rapports avec les autres personnages: "Une fille qu'a faite la vie pendant dix ans, ça poigne pus! Chus finie! Pis essayez donc d'expliquer ça à mes sœurs» ou encore: «Dix ans! Dix ans pour rien! C'est pas assez pour se tuer, ça, vous pensez ?»

Le $D_{2}$ extra. peut encore contribuer à préciser la vraisemblance du drame malgré les aspects invraisemblables de la technique scénique. Ainsi dans le monologue d'Angéline Sauvé où le $D_{2}$ extra. permet l'aveu qui rend la conduite d'Angéline vraisemblable: «Tout le monde peut pas avoir du fun dans les clubs, mais moé j'aime ça! C'est ben sûr que c'est pas vrai que j'prends juste un coke quand j'vas là! C'est ben sûr que j'prends d'la boisson!"

Une autre fonction du $D_{2}$ extra. consiste à faire progresser l'action dramatique particulièrement dans les cas où $D_{2}$ extra. et personnage-Destinataire intradiégétique ne font qu'un. Enfin l'étude du $D_{2}$ extra. nous renseigne sur une autre de ses fonctions qui consiste à manifester son accord ou son désaccord avec le message de l'œuvre et donc en quelque sorte à être le porte-parole de la morale de la pièce. Ainsi, dans les Belles-Sœurs, le ton dépouillé des monologues, la simplicité des phrases qui se résument souvent à des interjections de désespoir ou de profond malheur, l'isolement scénique qui marque bien la triste solitude des personnages-Destinateurs révèlent un $D_{2}$ extra. peu enclin à la condamnation mais au contraire compatissant voire même solidaire.

Reprenant au profit du théâtre ce qu'écrivait Gérald Prince ${ }^{31}$ au sujet du narrataire et de la narration, il faudrait affirmer que le $D_{2}$ extra. est un des éléments nécessaires de tout le système dramatique, que l'étude rigoureuse de ce qu'il représente dans le processus intentionnel de communication comme décodeur des signes qui lui sont adressés permet une lecture plus précise et mieux définie de l'œuvre.

Concluons brièvement. La recherche en sémiologie de l'œuvre théâtrale en est encore à ses premiers pas comme probablement la sémiologie de la langue elle-même qui en est son fondement. Plusieurs notions restent encore à préciser, plusieurs erreurs de méthode devront peut-être être corrigées, plusieurs intuitions devront être vérifiées. II nous semble, cependant, qu'en comparant la dramaturgie à une polysémie, à un système de signes qui est aussi processus intentionnel de communication et non seulement un moyen de signification ou de stimulation, nous nous donnons la possibilité d'entreprendre une étude méthodique de cette "machine infernale», encore mystérieuse, mais dont certains éléments et les lois qui les régissent ne nous échappent plus.

31. Gérald Prince, op. cit., p. 196. 
TABLEAU la

Signes dramatiques, graphes et fonctions

Encore cinq minutes de Françoise Loranger

ACTES/SÉQ. RÉGIES PERSONNAGES RÉPLIQUES

DÉCOR

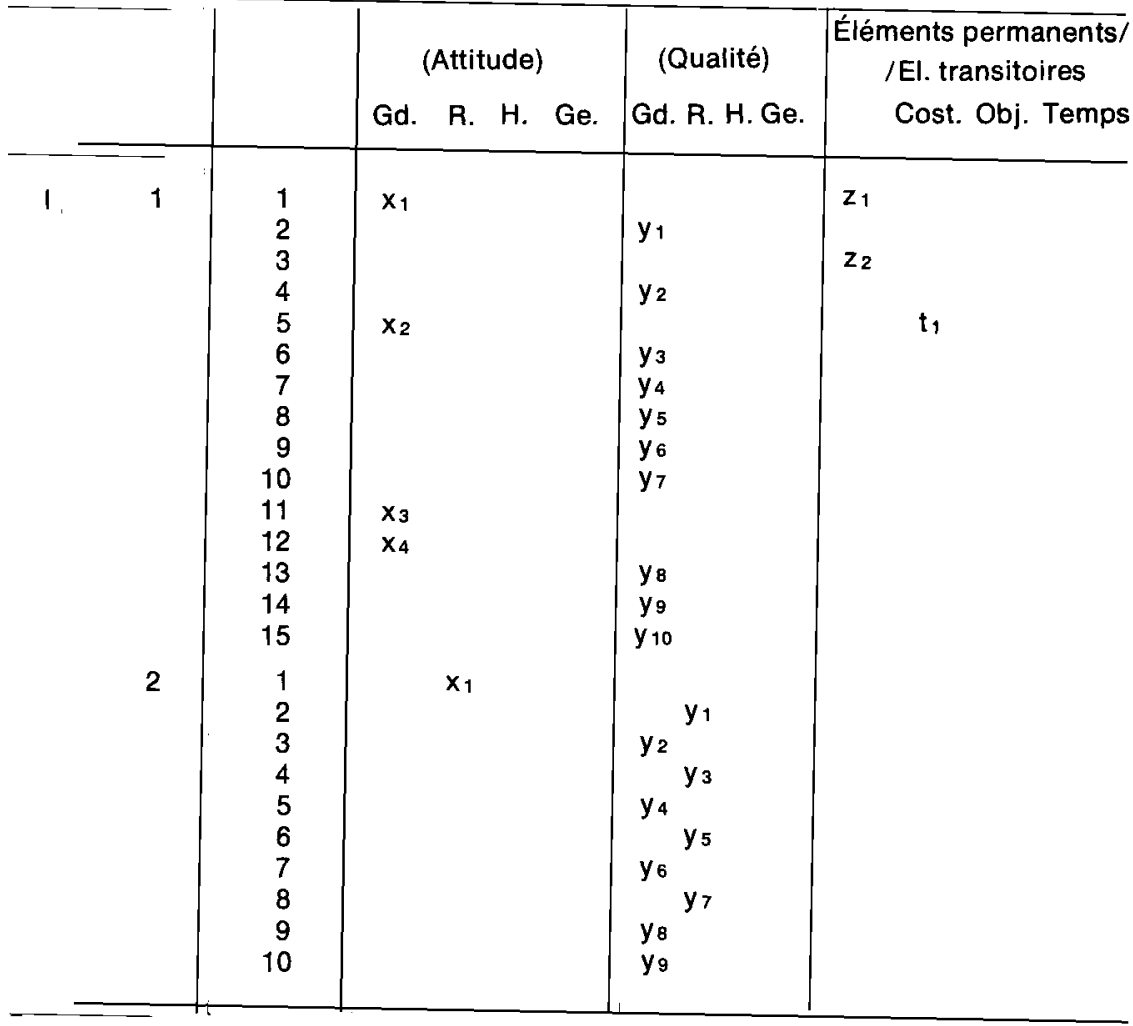

1. Personnages (attitude): nous regroupons ici tous les signes qui marquent des mouvements scéniques, des gestes, des mimiques du visage.

2. Répliques (qualité): nous plaçons sous cette rubrique, non pas le contenu de la réplique, mais sa qualité d'intonation, de tonalité, ("vivement», “avec angoisse")

3. Décor: Eléments permanents: lieux ou accessoires qui demeurent inchangés durant toute la pièce. Eléments transitoires: à ceux déjà indiqués, costumes, objects, temps, on devra selon les besoins de la description ajouter la musique, les bruits, l'éclairage, le maquillage, la coiffure, etc.

Légende :

Gd: Gertrude

R: Renaud

$H$ : Henri

Ge: Geneviève

$X$ : lettre choisie arbitrairement pour désigner tous les signes de la catégorie «Personnages" et l'exposant indique l'ordre d'apparition du signe dans cette même catégorie. II en va ainsi pour y (Répliques), z (Décor permanent), t (Costumes), etc. 


\section{TABLEAU Ib}

\begin{tabular}{|llllllll}
\hline$G$ & $R$ & $A$ & $P$ & $H$ & $E$ & $S$ \\
\hline
\end{tabular}

$$
\begin{aligned}
& P \rightarrow G d .+z_{1}+z_{2}+x_{1}+y_{1} \\
& S N \rightarrow G d .+z_{1}+z_{2} \\
& S V \rightarrow x_{1}+y_{1}
\end{aligned}
$$$$
P \rightarrow G d .+y_{2}+x_{2}+y_{3}+y_{4}+t_{1}+z_{1}
$$$$
\mathrm{SN} \rightarrow \mathrm{Gd} .+\mathrm{z}_{1}
$$$$
S V \rightarrow y_{2}+x_{2}+y_{3}+y_{4}+t_{1}
$$$$
P \rightarrow G d .+z_{1}+y_{5}+y_{6}+y_{7}+y_{8}
$$$$
+y_{9}+y_{10}+x_{3}+x_{4}
$$$$
\mathrm{SN} \rightarrow \mathrm{Gd} .+\mathrm{z}_{1}+\mathrm{y}_{5}+\mathrm{y}_{6}
$$$$
S V \rightarrow x_{3}+x_{4}+y_{7}+y_{8}+y_{9}+y_{10}
$$

$P \rightarrow G d .+y_{2}+y_{4}+y_{6}+z_{1}$

$\mathrm{SN} \rightarrow \mathrm{Gd} .+\mathrm{z}_{1}$

$S V \rightarrow y_{2}+y_{4}+y_{6}$

$P \rightarrow R+x_{1}+y_{1}+y_{3}+y_{5}$

$\mathrm{SN} \rightarrow \mathrm{R}$

$S V \rightarrow x_{1}+y_{1}+y_{3}+y_{5}$

\section{Légende:}

\section{$P:$ phrase}

SN: syntagme nominal

SV: syntagme verbal

N: nom

D: déterminant<smiles>[Y19]N[Al]SNPO[Z12]</smiles>

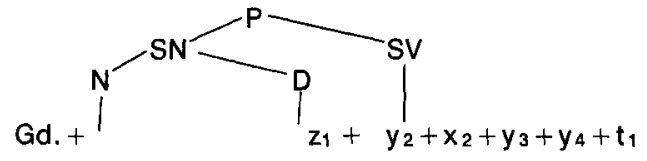

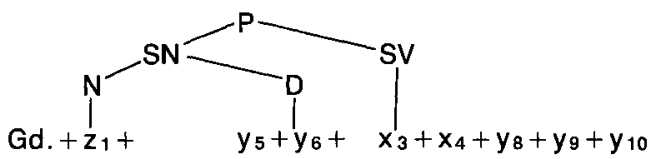

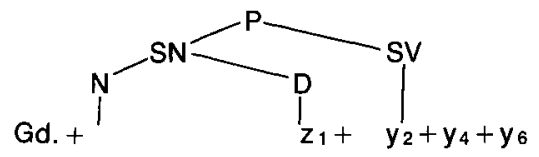<smiles>[Y19][Y9][Y19][As]P[Nb]([2H])[PH]</smiles> 
TABLEAU IC

\begin{tabular}{|c|c|c|c|c|c|c|c|c|}
\hline$F$ & 0 & $N$ & $\mathrm{C}$ & $\mathrm{T}$ & 1 & 0 & $N$ & $S$ \\
\hline
\end{tabular}

Perspective de Gertrude

Perspective de Renaud

DÉGRADATION POSSIBLE

$\left.\right|_{\text {PROCESSUS DE }}$

DÉGRADATION = AMÉLIORATION POSSIBLE
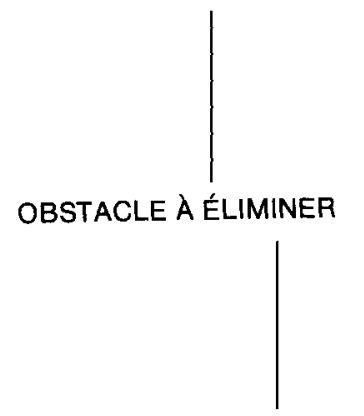

PÉRIL A ÉCARTER VS DOMMAGE A INFLIGER 


\section{AXES SÉMANTIQUES}

\section{TABLEAU II}

\begin{tabular}{|c|c|c|c|c|c|c|c|c|c|c|c|c|c|c|c|c|c|c|}
\hline \multirow{2}{*}{$\mathrm{P}$} & \multicolumn{2}{|c|}{$\begin{array}{l}\text { Motivation } \\
\text { psycho- } \\
\text { logique }\end{array}$} & \multicolumn{4}{|c|}{ Amour } & \multicolumn{4}{|c|}{ Sexualité } & \multicolumn{4}{|c|}{ Morale } & \multicolumn{4}{|c|}{$\begin{array}{l}\text { Rapports de } \\
\text { parenté surestimés } \\
\text { et sous-estimés }\end{array}$} \\
\hline & $a$ & $\mathrm{~b}$ & $\dot{a}$ & b & $c$ & $\mathrm{~d}$ & $a$ & b & $c$ & $d$ & $a$ & $b$ & $\mathrm{c}$ & $d$ & $a$ & $b$ & $\mathrm{c}$ & d \\
\hline AMÉLIE & $\phi$ & $\phi$ & $\phi$ & $\phi$ & $\phi$ & $\phi$ & $\phi$ & $\phi$ & $\phi$ & $\phi$ & $\phi$ & $+c$ & $+\mathbf{n}$ & $\phi$ & $\phi$ & $+u$ & $+o$ & $\phi$ \\
\hline ACHILLE & + & + & $\phi$ & $\phi$ & $\phi$ & $\phi$ & $\phi$ & $\phi$ & + & $\phi$ & $\phi$ & $+c$ & $\phi$ & $+n$ & $\phi$ & $+u$ & +0 & $\phi$ \\
\hline ÉLIZABETH & + & + & + & $\phi$ & + & $\phi$ & $\phi$ & $\phi$ & + & $\phi$ & $\phi$ & $\begin{array}{l}+c \\
+n\end{array}$ & $\begin{array}{l}+c \\
+n\end{array}$ & $\phi$ & $\phi$ & $\begin{array}{l}+u \\
+0\end{array}$ & $\begin{array}{l}+u \\
+0\end{array}$ & $\phi$ \\
\hline ROBBERT & + & + & $\phi$ & $\phi$ & $\phi$ & $\phi$ & + & $\phi$ & $\phi$ & $\phi$ & $\phi$ & $+n$ & $+n$ & $\phi$ & $\phi$ & $\begin{array}{l}+u \\
+0\end{array}$ & & $\phi$ \\
\hline GENEVIÈVE & + & + & $\phi$ & + & $\phi$ & + & $\phi$ & $\phi$ & + & $\phi$ & $\phi$ & $+n$ & $\phi$ & $+n$ & $\phi$ & $\begin{array}{l}+\mathrm{u} \\
+\mathrm{o}\end{array}$ & $\begin{array}{l}+u \\
+o\end{array}$ & $\phi$ \\
\hline LAURA & $\phi$ & + & $\phi$ & $\phi$ & $\phi$ & $\phi$ & $\phi$ & + & $\phi$ & $\phi$ & $\phi$ & $+n$ & $\phi$ & $\phi$ & $\phi$ & $\phi$ & $\phi$ & $\phi$ \\
\hline RICHARD & $\phi$ & $\phi$ & $\phi$ & + & $\phi$ & $\phi$ & + & $\phi$ & $\phi$ & $\phi$ & $\phi$ & $+n$ & $\phi$ & $\phi$ & $\phi$ & $\phi$ & $\phi$ & $\phi$ \\
\hline MANON & $\phi$ & $\phi$ & $\phi$ & $\phi$ & $\phi$ & $\phi$ & + & $\phi$ & $\phi$ & $\phi$ & $\phi$ & $\phi$ & $\phi$ & $\phi$ & $\phi$ & $\phi$ & $\phi$ & $\phi$ \\
\hline
\end{tabular}

\section{Légende: $\quad F$ : axe sémantique fonctionnel $\phi:$ absence de fonction}

Motivation psychologique: $a=$ événement de l'enfance; $b=$ événement important de la vie adulte

Amour, Sexualité, Morale, Rapports de parenté: $a=$ qualification unique; $b=$ plusieurs qualifications; $c=a c t e$ unique;

$d=$ plusieurs actes

Morale : $+c=$ respect de la morale conventionnelle $+n=$ non respect de la morale conventionnelle

Rapports de parenté: $+\mathrm{u}=$ rapports de parenté surestimés; +o = rapports de parenté sous-estimés 


\section{TABLEAU III}

\section{Le modèle hexagonal}

SYSTÈME DE LA SEXUALITÉ

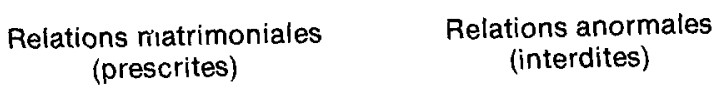

(prescrites)

(interdites)

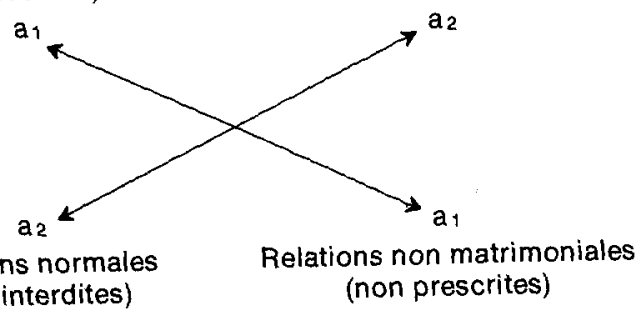

Relations normales

(non prescrites)

Rel. sex. profitables

$b_{1}$

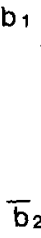

$\bar{b}_{2}$

Rel. sex. non nuisibles
Rel. sex. nuisibles

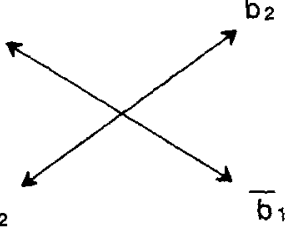

Rel. sex. non profitables
Rel. sex.

désirées

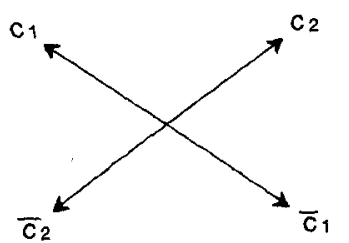

Rel. sex. non craintes
Rel. sex.

craintes

Rel. sex. non désirées

Achille $\rightarrow$ Élizabeth: $a_{1}+b_{1}+\bar{c}_{1}=$ relations sexuelles conflictuelles dont le manque sera comblé par

Achille $\rightarrow$ Laura: $c_{1}+\bar{b}_{2}+\bar{a}_{2}=$ relations sexuelles compatibles

\section{SYSTĖME DE LA MORALE}

Rel. morale

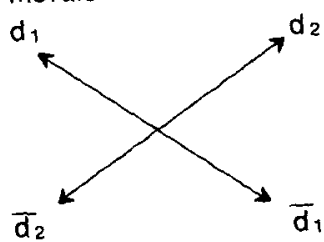

SYSTEME DE L'AMOUR

Rel. d'amour Rel. de crainte

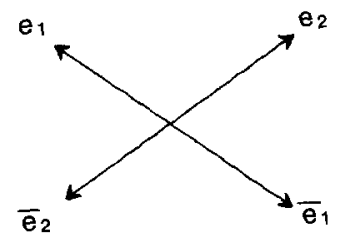

Rel. de non amour

Rel. de non haine

Rel. non amorale

Rel. non morale

Achille $\rightarrow$ Élizabeth: $d_{1}+\bar{e}_{1}=$ relation conflictuelle entre la morale et l'amour 


\section{TABLEAU IV \\ Les syntagmes narratifs dans $A u$ retour des oies blanches de Marcel Dubé}

\section{PREMIER TABLEAU: QUALIFICATION DU HÉROS-SUJET}

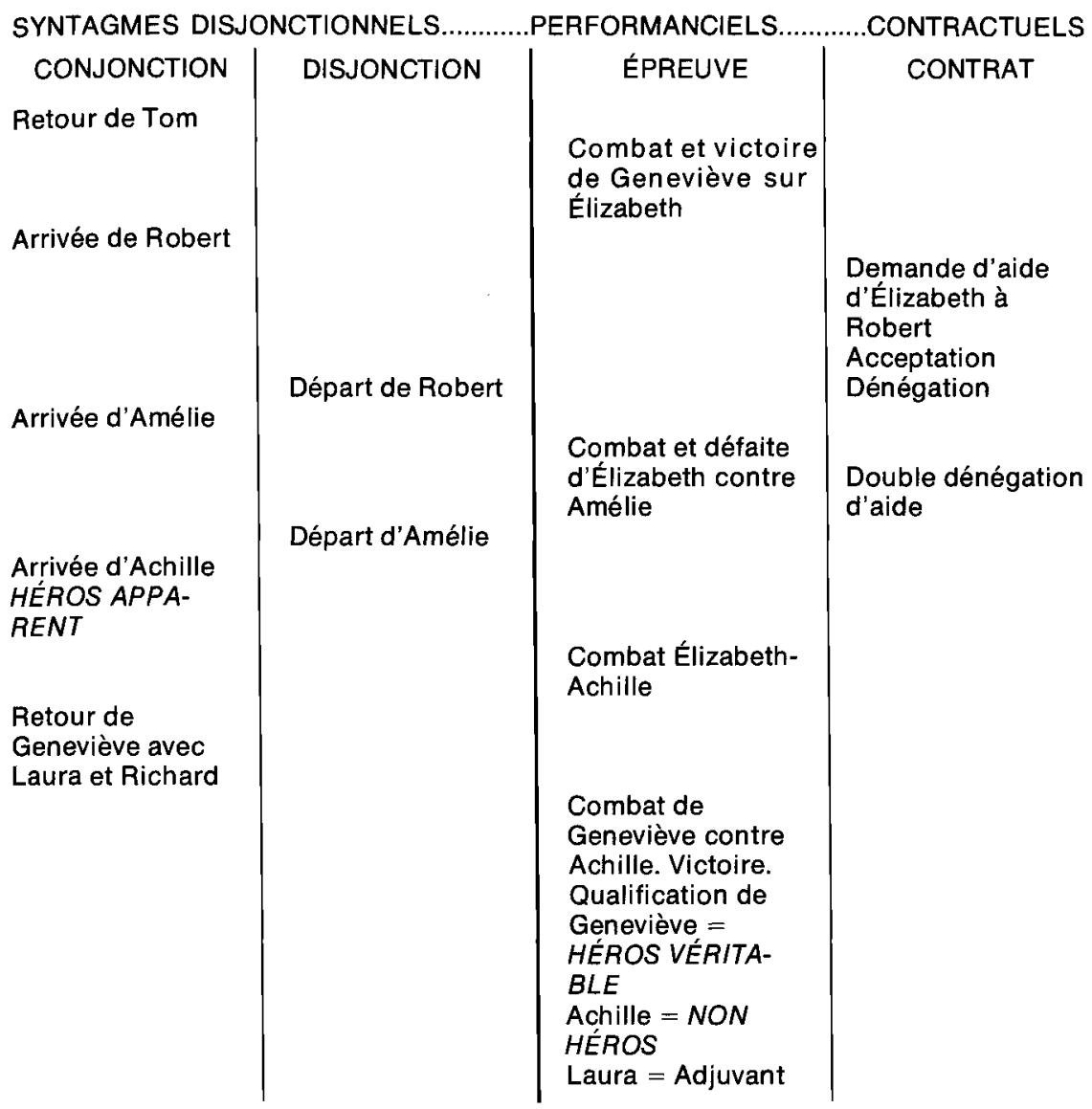

DEUXIÈME TABLEAU: CONFRONTATION OU ÉPREUVE PRINCIPALE

\begin{tabular}{l|l|l} 
& $\begin{array}{l}\text { Départ de Gene- } \\
\text { viève pour aller se } \\
\text { changer }\end{array}$ & \\
& & $\begin{array}{l}\text { Mandement: } \\
\text { Laura } \\
\text { Acceptation : } \\
\text { Robert }\end{array}$ \\
$\begin{array}{l}\text { Retour de Gene- } \\
\text { viève au salon }\end{array}$ & $\begin{array}{l}\text { Combat et échec } \\
\text { de Laura }\end{array}$ & $\begin{array}{l}\text { Mandement : } \\
\text { Geneviève } \\
\text { Dénégation : } \\
\text { Robert }\end{array}$
\end{tabular}


Retour d'Achille

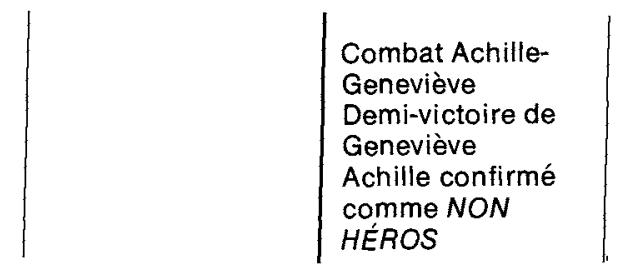

TROISIĖME TABLEAU: VICTOIRE DU HÉROS - MANQUE COMBLÉ
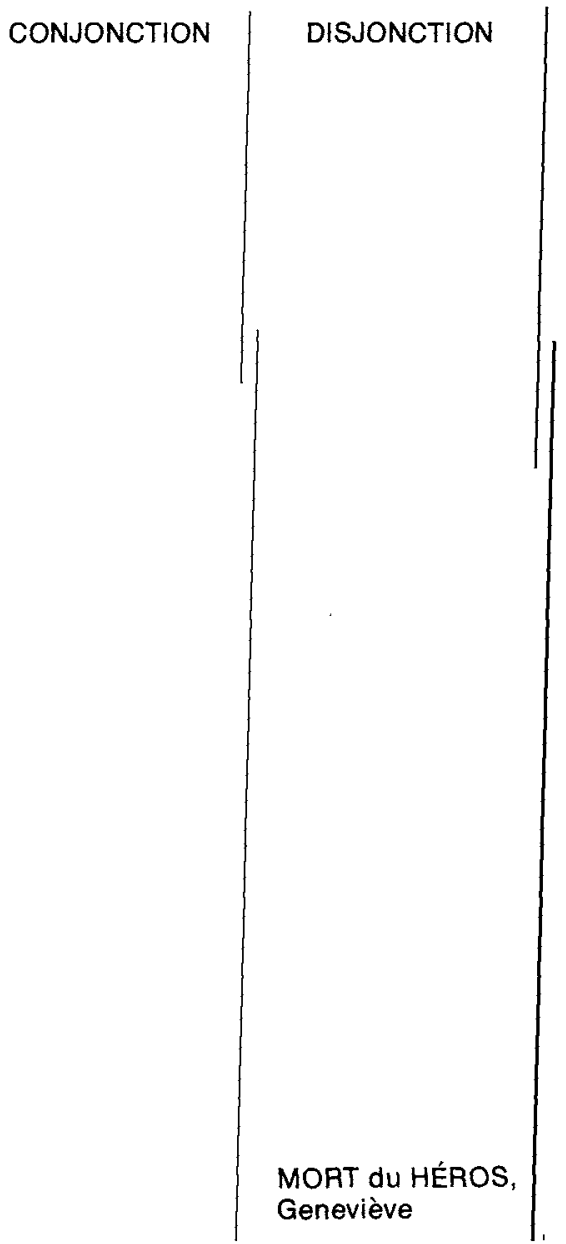

ÉPREUVE

CONTRAT

Geneviève se donne la mission de venger Tom

Combat de GeneAcceptation Amtre Achille, Amélie, Élizabeth

Épreuve qualifiante: réception d'aide de Laura: qualification du HÉROS SUJET Combat et victoire de Geneviève sur Achille

Combat: Geneviève vs Amélie Combat: Geneviève vs Élizabeth

MORT dU HÉROS, Geneviève

Combat et victoire de Geneviève sur Élizabeth

QUATRIÈME TABLEAU: SÉQUENCE FINALE

Folie d'Élizabeth

Mandement:

Achille

Acceptation:

Amélie

Achille devient à nouveau HÉROS APPARENT 